\title{
Evaluación de la aplicación del Índice de Inclusión en la Facultad de Psicología de la Universidad de Cuenca, Ecuador
}

\section{Assessment of the application of the Index for Inclusion in the Faculty of Psychology of the University of Cuenca, Ecuador}

\author{
Ruth Clavijo $^{1}$ (D), Freddy Cabrera ${ }^{2}$, Ángel Japón ${ }^{2}$ \\ ${ }^{1}$ Facultad de Psicología, Universidad de Cuenca, Avenida 12 de abril y Loja, Cuenca, Ecuador. \\ ${ }^{2}$ Facultad de Filosofía, Universidad de Cuenca, Avenida 12 de abril y Loja, Cuenca, Ecuador \\ Autor para correspondencia: ruth.clavijo@ucuenca.edu.ec \\ Fecha de recepción: 28 de marzo de 2020 - Fecha de aceptación: 16 de abril de 2020
}

\section{RESUMEN}

El reconocimiento del derecho al acceso, permanencia y culminación de la educación superior sin ningún tipo de discriminación supone un avance hacia un modelo de educación sostenible e incluyente. La educación inclusiva tiene que ver con el proceso de reducir las barreras que limitan la presencia y la participación de los estudiantes, procurando eliminar todo tipo de formas que conlleven a la exclusión. Las universidades, aunque diversas en sus prioridades, precisan avanzar hacia un modelo de educación basado en los principios de la educación inclusiva. El propósito del presente estudio consistió en identificar la percepción de docentes y estudiantes sobre culturas, políticas y prácticas inclusivas de la Facultad de Psicología de la Universidad de Cuenca en Ecuador. Para ello se utilizó una muestra conformada por 241 estudiantes y 44 docentes. Las percepciones de docentes y estudiantes con respecto a la educación inclusiva fueron recolectadas utilizando la guía del Índice de Inclusión y los datos fueron procesados mediante estadísticos descriptivos e inferenciales no paramétricos. Los resultados revelaron que, tanto estudiantes como profesores, están de acuerdo en las tres dimensiones evaluadas. Se encontró que la dimensión crear culturas inclusivas es la más valorada, seguida de la dimensión desarrollar prácticas inclusivas, y finalmente, la menos valorada fue la dimensión elaborar políticas inclusivas. Además, es importante señalar que en promedio $8.4 \%$ de los ítems del Índice de Inclusión presentaron valores inusuales debido a falta de información, lo que da lugar al planteamiento de un estudio de validez del constructo del Índice para la Inclusión.

Palabras clave: Educación superior, cultura, políticas y prácticas inclusivas.

\begin{abstract}
The recognition of the right to access, reside and complete higher education without any type of discrimination, represents a move towards a model of sustainable and inclusive education. Inclusive education refers to the process of reducing the barriers that limit the presence and participation of students, trying to eliminate all kinds of ways that lead to exclusion. Universities, although diverse in their priorities, need to move towards an education model based on the principles of inclusive education. The purpose of this study consisted in identifying the perception of teachers and students about cultures, policies, and inclusive practices of the Faculty of Psychology, of the University of Cuenca in Ecuador. For this matter, a sample consisting of 241 students and 44 teachers was used. The perception of docents and students regarding inclusive education was collected using the guide Index for Inclusion and the data were processed by descriptive and inferential non-parametric statistics. The results revealed that both, students and teachers, agree on the three evaluated dimensions. It was found that the dimension to create inclusive cultures is the highest valued, followed by the dimension to develop inclusive practices, and finally, the least valued was the dimension to elaborate on inclusive policies. Moreover, it is important to point out that on average $8.4 \%$ of the items of the Index of Inclusion presented unusual values due to lack of information, which gives rise to the proposal of conducting a validity study of the construct of the Index for Inclusion.
\end{abstract}

Keywords: Higher education, culture, policies and inclusive practices.

\section{INTRODUCCIÓN}

La inclusión educativa es uno de los principales temas que se encuentra agendado dentro de los Objetivos de Desarrollo Sostenible (ODS) 2030, haciéndose visible a través del objetivo 4 que contempla: "Garantizar una educación inclusiva, equitativa y de calidad y promover oportunidades de aprendizaje durante toda la vida para todos" (UNESCO, 2016. p.5); en donde se destaca sobre todo a la inclusión e igualdad como pilares fundamentales para asegurar una enseñanza de calidad. De igual manera, 
la Organización de las Naciones Unidas para la Educación, la Ciencia y la Cultura (UNESCO) a través de sus planteamientos fomenta el desarrollo de sistemas de educación inclusivos, respaldando políticas, programas y prácticas en materia de educación.

Por otro lado, la consideración de los Derechos Humanos (Naciones Unidas, 2015a) ha facilitado el cuestionamiento al tratamiento general que se ha venido otorgando a colectivos minoritarios, personas con discapacidad, indígenas, mujeres, sectores que históricamente han sido excluidos de la educación formal (Herdoíza, 2015). Actualmente, los cambios en nuestras sociedades han facilitado la visibilidad de estos grupos y de las personas que los conforman, resaltando la enorme inequidad e injusticia que supone no sumar esfuerzos necesarios, desde la formulación de políticas públicas emitidas por las instancias reguladoras que facilite el acceso efectivo de los estudiantes a los distintos niveles educativos, donde puedan realizar su proceso de aprendizaje con absoluta participación y respeto de sus diferencias (Ainscow, Booth, \& Dyson, 2006; Cotán, 2017).

Existen a nivel internacional y regional, organismos como la UNESCO, la Comisión Económica para América Latina (CEPAL), la Oficina Regional de Educación para América Latina y el Caribe (OREALC) entre otras instancias, que influyen en la generación de políticas, motivando a los estados a garantizar la educación como un derecho humano, sugiriendo a las universidades la responsabilidad social de convertirse en agentes activos en el cumplimiento de estas políticas (Fernández, 2013; Fernández \& Pérez, 2016; Sinchi \& Gómez, 2018). Las universidades deberán examinar su rol, revisar su misión y visión institucional, reevaluar sus funciones sustanciales, reduciendo las barreras que dificultan el aprendizaje, la presencia y la participación de todos los estudiantes, rescatando las competencias que le corresponde como Instituciones de Educación Superior (IES), en la formación de profesionales, respetuosos de la diversidad donde el reconocimiento, la aceptación y el apoyo de las diferencias, sea una constante (Echeita \& Navarro, 2014; González, González, Guerrero, \& Ríos, 2018).

Durante los últimos tiempos, varios son los compromisos asumidos por algunos organismos internacionales para combatir las desigualdades dentro de los países, procurando construir ciudades pacíficas, justas e inclusivas, proteger los derechos humanos, promover la igualdad entre los géneros, buscando el empoderamiento de las mujeres y las niñas en todos los espacios de participación, garantizando una protección del derecho a la educación y creando las condiciones necesarias para un crecimiento económico sostenible e inclusivo (Naciones Unidas, 2015b); sin embargo, en la consecución de estos objetivos la universidad, como parte de su responsabilidad social, tiene una gran tarea pendiente (Fernández, 2013; Aznar \& Barrón, 2017; Cotán, 2017). La universidad requiere trascender sus fronteras impulsando cambios sociales, promoviendo el respeto y la tolerancia para una mejor convivencia (González et al., 2018). Las dinámicas sociales de la actualidad exigen una universidad inclusiva que de la bienvenida a la diversidad a partir del respeto de las diferencias, brindando a todos la oportunidad de ser apoyados y aceptados (Espinosa, Gómez, \& Cañedo, 2012).

En la actualidad, las aulas universitarias parecen evidenciar cada vez más la diversidad dentro de ellas, y aunque pareciera que siempre han sido diversas, en los últimos años, factores como: la movilidad estudiantil, el acceso de mayor diversidad de estudiantes, la presencia de un porcentaje alto de mujeres y grupos minoritarios, las mayores oportunidades de ingreso de estudiantes con discapacidad, entre otras, convierten a las aulas en espacios altamente heterogéneos, situación que conlleva un desafío para el profesorado que se encuentra preocupado por conocer y defender las diferencias y promover una educación sin barreras (Leire, Fernández, \& Goicoechea, 2010; Moriña, Cortés, \& Molina, 2015; Ochoa Cervantes, 2019).

En el Ecuador, el Sistema de Educación Superior, por mandato constitucional, se encuentra articulado al Plan Nacional de Desarrollo (PND), el mismo que considera como principios orientadores entre otros: la autonomía responsable, el cogobierno, la igualdad de oportunidades, la calidad y pertinencia (Herdoíza, 2015; Naciones Unidas, 2015b; SENPLADES, 2017). Alineada al PND, la inclusión educativa en los últimos años se ha convertido en una prioridad estatal (Asamblea Nacional, 2010). Para este propósito, se la ha enmarcado en normativas, leyes, reglamentos y ordenanzas, que procuran reducir barreras que excluyen al ser humano de la educación (Jara, Melero, \& Guichot, 2015). El logro de la equidad y la justicia social, el reconocimiento de las diferencias individuales y la mejora de las condiciones de participación de cada estudiante, son los derroteros que deben alumbrar el camino de las IES en todo el país.

El marco legal vigente de la educación superior (ES) en nuestro país, propone importantes cambios para garantizar el acceso y la participación como un derecho fundamental. Particularmente importantes son dos acciones relacionadas con el acceso a la ES: por un lado, el principio de gratuidad y, por otro, la aplicación del examen de admisión denominado ENES, a partir del 2012 (Ponce \& Carrasco, 2017). La aplicación de la política de gratuidad pretendía garantizar el acceso a colectivos con escasos recursos, mientras que la implementación del examen de ingreso (de aptitudes), buscaba garantizar el acceso, la permanencia y culminación del proceso educativo (Ponce \& Carrasco, 2017). Paralelamente, el cierre de universidades que no cumplían criterios mínimos de calidad permitió incluir en los procesos de evaluación y acreditación de la IES, estándares relacionados con la educación inclusiva (Sinchi \& Gómez, 2018).

La Universidad de Cuenca, por su parte, propone medidas orientadas a eliminar toda forma de discriminación, ya sea basada en género, etnia, condición social y orientación sexual, así como garantizar la igualdad de los grupos tradicionalmente discriminados (Universidad de Cuenca, 2017). Contempla criterios para asegurar la inclusión, identificando barreras físicas, sociales, educativas y actitudinales que estén presenten en el campus y que dificulten la verdadera participación, todo esto en consonancia con la filosofía de inclusión que defiende una educación eficaz para todos (Leire et al., 2010; Moriña $e t$ al., 2015).

Sin embargo, a pesar de los avances legislativos realizados en Ecuador, consideramos que la educación inclusiva es un tema complejo que debe abordarse desde distintos ámbitos. La promulgación de leyes es una cuestión necesaria, pero no suficiente, debe ir acompañada de la voluntad de cada uno de los miembros de la comunidad universitaria a través de un trabajo sistematizado (Corral, Villafuerte, \& Bravo, 2015; Herdoíza, 2015; De la Hoz 
Blanco, 2017). Las medidas coordinadas deben reducir cualquier tipo de desigualdad o exclusión dentro de las IES, la finalidad última será generar una verdadera cultura inclusiva, es decir, construir una comunidad segura, acogedora, donde cada estudiante se sienta parte, respetado en su diversidad, en su unicidad (Booth \& Ainscow, 2002; Ortiz \& Lobato, 2003; Pardo, 2010). El camino por seguir para la transformación educativa en el Ecuador tiene aún un largo trecho que recorrer. Creemos que la formación académica en contextos universitarios no ha recibido la atención necesaria, tampoco existen instrumentos que permitan medir la educación inclusiva. La diversidad y el derecho a una educación equitativa requieren de preparación cultural, tanto individual como colectiva de los diferentes miembros de la comunidad universitaria (Cotán, 2017).

En este contexto, nos hemos propuesto conocer la percepción que tienen docentes y estudiantes de la Facultad de Psicología sobre la educación inclusiva a nivel superior, considerando que la ES constituye un derecho y un bien social que sienta las bases de una sociedad justa y más equitativa. La universidad es el contexto propicio para generar y promover una educación más inclusiva, mediante una comprensión básica de la diversidad, reflejada en las actividades profesionales de todos sus graduados, competentes para un futuro mejor y más digno para todos.

\section{MATERIALES Y MÉTODO}

\subsection{Instrumento}

La información de campo fue levantada por cuatro estudiantes de la Facultad de Psicología de la Universidad de Cuenca quienes evaluaron por separado dos bases de datos diferentes: una de los profesores $(n=44$, casos válidos) y otra de los estudiantes ( $n=241$, casos válidos) para sus respectivos trabajos de graduación (Loja \& Tello, 2019; Jara \& Narváez, 2019) en el marco del Proyecto de Investigación: Percepción de docentes y estudiantes sobre culturas, políticas y prácticas inclusivas en educación superior (Convocatoria DIUC, XVII, 2018), que los autores del presente artículo dirigen.

Para cumplir con el objetivo se ha utilizado el Index for Inclusion, propuesta adaptada por Salceda \& Ibáñez (2015), que deriva del instrumento original (Booth \& Ainscow, 2002) y que ha adaptado en dos ocasiones, y traducido no solamente al español, sino a más de 10 idiomas. La primera, dirigida para Educación Infantil (González-Gil, Gómez-Vela, \& Jenaro, 2007) y la segunda para Educación Primaria (Durán et al., 2002), estas dos versiones con acceso abierto y gratuito. La tercera edición del "Index for Inclusion" (Booth, Simón, Sandoval, Echeita, \& Muñoz, 2015) incorpora, entre otras innovaciones, la sostenibilidad al análisis actual del currículo escolar, que es tan necesario para la investigación. La razón principal para aplicar esta herramienta radica en que el instrumento es internacionalmente reconocido, además permite evaluar el proceso de educación inclusiva en los diferentes escenarios, dónde toda la comunidad educativa puede

\footnotetext{
${ }^{1}$ Según el Reglamento de Carrera y Escalafón Docente del profesor e investigador de la Universidad de Cuenca, los docentes titulares ingresan mediante concurso público de
}

contribuir a combatir cualquier forma de exclusión promoviendo culturas, políticas y prácticas inclusivas (Booth et al., 2015). El instrumento tiene cuatro opciones de respuesta para cada ítem, tres de ellas pertenecen a una escala (1=En desacuerdo, 2=Bastante de acuerdo, $3=$ Totalmente de acuerdo) y la cuarta alude al hecho de que el estudiante o profesor necesita más información para responder el cuestionario.

\subsection{Análisis de los datos}

Los datos fueron procesados en el software SPSS 24 (Martínez, Castellanos, \& Chacón, 2015), y se presentan mediante estadísticos descriptivos e inferenciales. Los estadísticos descriptivos constituyen las frecuencias y porcentajes, así como las medias y desviaciones estándar de cada uno de los ítems del instrumento. Para verificar la fiabilidad del instrumento se empleó el coeficiente Alpha de Cronbach mismo que obtuvo un valor de 0.954 equivalente a una alta fiabilidad; al separar los resultados de los estudiantes y profesores se encontró que el alfa de los profesores es de $0.953 \mathrm{y}$ el de los estudiantes de 0.956 . Debido a que los promedios de las dimensiones no cumplen con el supuesto de normalidad, se empleó la prueba no paramétrica U de Mann-Whitney para comparar las dimensiones entre el grupo de estudiantes y el grupo de profesores (excepcionalmente se empleó t de Student cuando se demuestra distribución normal). Adicionalmente, se compararon las seis secciones del Índex empleando la prueba no paramétrica KruskalWallis, con la cual se determinó que existen diferencias significativas entre las dimensiones $(p<0.05)$. Para identificar qué diferencias específicamente existen, al comparar cada sección con las otras cinco, se empleó U de Mann-Whitney a manera de análisis post hoc (Ruxton \& Beauchamp, 2008). Con la prueba señalada se puede calcular Z, si este valor tiene un valor negativo mayor que $1.96(\mathrm{Z}<-1.96)$, se rechaza la hipótesis nula y se acepta la hipótesis de las diferencias.

\subsection{Participantes}

En la Tabla 1 se exhibe la información del perfil de los estudiantes $(n=241)$ y profesores $(n=44)$ que participaron como muestra probabilística por conglomerado en la Facultad de Psicología. Los estudiantes en su mayoría son mujeres $(65.6 \%)$, en el caso de los profesores, el son hombres $(61.4 \%)$. Consultando sobre la etnia, la mayoría de las estudiantes y profesores se identifican como mestizos (94 y 91\%, respectivamente). La edad de los estudiantes oscila entre los 20 a los 25 años; en el caso de los docentes, al menos la mitad se encuentra en el intervalo de 30 a 39 años. El $65 \%$ de estudiantes procede de la provincia del Azuay, seguido de la provincia del Cañar. El 96.7\% de estudiantes señaló no tener discapacidad, sin embargo, hay quienes presentan discapacidad visual, física y psicosocial. La mitad de los docentes no ha recibido capacitación en educación inclusiva y en su mayoría (61.4\%) no tiene más de 5 años de experiencia docente. Con respecto a la situación laboral, el $68.2 \%$ es profesor ocasional, únicamente el $20.5 \%$ es profesor titular ${ }^{1}$. En cuanto al nivel de estudios de los profesores,

méritos y oposición, se clasifican en: principales, agregados y auxiliares y la condición de titular garantiza estabilidad (p.2). 
Tabla 1. Perfil de los estudiantes que participaron del estudio.

\begin{tabular}{|c|c|c|c|c|c|}
\hline \multirow{2}{*}{ Variable } & \multirow{2}{*}{ Valor final } & \multicolumn{2}{|c|}{ Estudiantes } & \multicolumn{2}{|c|}{ Profesores } \\
\hline & & Frecuencia & Porcentaje & Frecuencia & Porcentaje \\
\hline \multirow{2}{*}{ Género } & Hombre & 83 & 34.4 & 27 & 61.4 \\
\hline & Mujer & 158 & 65.6 & 17 & 38.6 \\
\hline \multirow{3}{*}{ Etnia } & Mestizo & 227 & 94.2 & 40 & 90.9 \\
\hline & Blanco & 10 & 4.1 & 3 & 6.8 \\
\hline & Indígena u otro & 4 & 1.7 & 1 & 2.3 \\
\hline \multirow{6}{*}{ Edad $^{\mathrm{a}}$} & 20-22 años & 98 & 40.7 & & \\
\hline & 23-25 años & 113 & 46.9 & & \\
\hline & 26-29 años & 21 & 8.8 & 9 & 20.5 \\
\hline & 30-39 años & 8 & 3.3 & 22 & 50.0 \\
\hline & 40-49 años & 1 & 0.1 & 8 & 18.2 \\
\hline & 50-59 años & & & 5 & 11.4 \\
\hline \multirow{6}{*}{ Procedencia } & Azuay & 158 & 65.6 & & \\
\hline & Cañar & 34 & 14.1 & & \\
\hline & Amazonía & 7 & 2.9 & & \\
\hline & Costa & 12 & 5.0 & & \\
\hline & Resto de la sierra & 13 & 5.4 & & \\
\hline & Extranjero & 2 & 0.8 & & \\
\hline \multirow{4}{*}{ Discapacidad } & Sin discapacidad & 233 & 96.7 & & \\
\hline & Visual & 4 & 1.7 & & \\
\hline & Física & 3 & 1.2 & & \\
\hline & Psicosocial & 1 & 0.4 & & \\
\hline \multirow{2}{*}{$\begin{array}{l}\text { Capacitación en } \\
\text { educación inclusiva }\end{array}$} & No & & & 22 & 50.0 \\
\hline & $\mathrm{Si}$ & & & 22 & 50.0 \\
\hline \multirow{3}{*}{ Experiencia docente } & 0-5 años & & & 27 & 61.4 \\
\hline & 6-10 años & & & 10 & 22.7 \\
\hline & 11 o más años & & & 7 & 15.9 \\
\hline \multirow{3}{*}{ Situación laboral } & Titular & & & 9 & 20.5 \\
\hline & Ocasional & & & 30 & 68.2 \\
\hline & Técnico docente & & & 5 & 11.4 \\
\hline \multirow{3}{*}{ Nivel de estudios } & Tercer nivel & & & 3 & 6.8 \\
\hline & Maestría & & & 37 & 84.1 \\
\hline & Doctorado & & & 4 & 9.1 \\
\hline
\end{tabular}

el $84.1 \%$ ha obtenido el cuarto nivel con maestría, mientras que, únicamente el $9.1 \%$ ha conseguido el nivel de doctorado.

\section{RESULTADOS}

\subsection{Evaluación de los ítems en las 6 secciones del Índice de Educación Inclusiva para las participantes}

Se han organizado en tres dimensiones, cada una con dos secciones como se estructura en el Index for Inclusion. La Dimensión A: Crear culturas inclusivas en la que se incluyen las secciones A.1. Construir comunidad y A.2. Establecer valores inclusivos. La Dimensión B: Elaborar políticas inclusivas que incluye: B.1. Desarrollar una universidad para todas las personas y B.2. Organizar el apoyo para atender a la diversidad. Finalmente, se presenta a la Dimensión C: Desarrollar prácticas inclusivas que contiene: C.1. Orquestar el proceso educativo y C.2. Movilizar recursos. Cada una de estas dimensiones y secciones se presentan por frecuencias y porcentajes para conocer el comportamiento general. Los resultados para cada uno de los ítems que pertenecen a las secciones se presentan en la Tabla 2.

En la primera sección: Construir comunidad, destacan en la escala quienes están 'en desacuerdo' en ítems como Todo el mundo se siente incluido, en el que se muestran en desacuerdo casi la mitad (48.8\%), seguido por el ítem $L a$ facultad involucra a la comunidad universitaria en el diseño de propuestas para la mejora de la convivencia interna $(41.1 \%)$. 
Table 2. Distribución porcentual de la evaluación de los participantes sobre los ítems de las 6 secciones del Índice de inclusión.

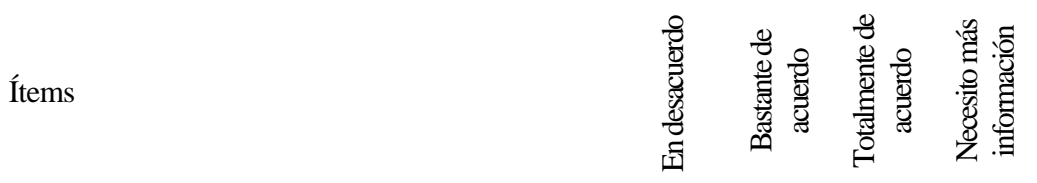

\begin{tabular}{|c|c|c|c|c|c|}
\hline \multicolumn{6}{|c|}{ A.1. Construir comunidad } \\
\hline A.1.1 & Todo el mundo se siente acogido. & 48.8 & 35.4 & 5.6 & 10.2 \\
\hline A.1.2 & Los recursos del entorno local se utilizan para apoyar el aprendizaje. & 33.7 & 56.8 & 5.3 & 4.2 \\
\hline A.1.3 & La facultad implementa estrategias para vincularse a redes locales y regionales de política social. & 21.1 & 61.8 & 11.9 & 5.3 \\
\hline A.1.4 & $\begin{array}{l}\text { La facultad involucra a la comunidad universitaria en el diseño de propuestas para la mejora de } \\
\text { la convivencia interna. }\end{array}$ & 41.1 & 43.5 & 7.0 & 8.4 \\
\hline A. 1.5 & El aula es un espacio social y educativo de participación. & 16.8 & 61.4 & 21.1 & .7 \\
\hline A.1.6 & $\begin{array}{l}\text { Existe colaboración entre el profesorado y el personal no docente (administrativo, consejería, } \\
\text { limpieza). }\end{array}$ & 24.6 & 51.2 & 16.5 & 7.7 \\
\hline A.1.7 & $\begin{array}{l}\text { El trabajo desarrollado por el personal no docente (administrativo, consejería, limpieza) es } \\
\text { conocido y valorado por estudiantes y profesorado. }\end{array}$ & 36.5 & 48.1 & 11.9 & 3.5 \\
\hline A.1.8 & Las relaciones entre el profesorado y el alumnado se basan en el respeto mutuo. & 8.8 & 63.9 & 26.7 & .7 \\
\hline A.1.9 & $\begin{array}{l}\text { El alumnado participa en los órganos de representación estudiantil (Consejo Directivo, Junta } \\
\text { académica, Consejo Universitario). }\end{array}$ & 18.6 & 51.2 & 25.6 & 4.6 \\
\hline A.1.10 & El alumnado se apoya mutuamente para lograr metas educativas. & 35.4 & 49.5 & 11.2 & 3.9 \\
\hline A.1.11 & Toda la comunidad universitaria siente orgullo de pertenecer a esta institución. & 17.9 & 49.1 & 23.9 & 9.1 \\
\hline \multicolumn{6}{|c|}{ A.2. Establecer valores inclusivos } \\
\hline A.2.1 & $\begin{array}{l}\text { Toda la comunidad universitaria se identifica con la filosofía, los principios y los objetivos de la } \\
\text { educación inclusiva. }\end{array}$ & 44.2 & 41.8 & 6.0 & 8.1 \\
\hline A. 2.2 & $\begin{array}{l}\text { La facultad transmite al entomo local que es una institución donde se generan transformaciones } \\
\text { que contribuyen a mejorar la sociedad. }\end{array}$ & 18.2 & 64.9 & 10.9 & 6.0 \\
\hline A. 2.3 & $\begin{array}{l}\text { La facultad impulsa acciones que promueven conductas sociales positivas y la solidaridad en } \\
\text { oposición al individualismo y el utilitarismo. }\end{array}$ & 23.9 & 55.4 & 14.4 & 6.3 \\
\hline A. 2.4 & La facultad implementa acciones para prevenir riesgos psicosociales. & 26.7 & 49.5 & 15.1 & 8.8 \\
\hline A. 2.5 & La facultad implementa acciones para disminuir las prácticas discriminatorias. & 28.4 & 51.2 & 14.0 & 6.3 \\
\hline A. 2.6 & $\begin{array}{l}\text { Todos los miembros de la facultad son tratados como personas que desempeñan un "rol" } \\
\text { fundamental para el buen funcionamiento de la misma. }\end{array}$ & 26.3 & 51.2 & 16.1 & 6.3 \\
\hline A. 2.7 & El profesorado tiene expectativas altas sobre todo el alumnado. & 28.8 & 48.4 & 15.8 & 7.0 \\
\hline A.2.8 & El profesorado implementa acciones para eliminar las barreras al aprendizaje y la participación. & 31.6 & 50.5 & 12.3 & 5.6 \\
\hline \multicolumn{6}{|c|}{ B.1. Desarrollar una universidad para todas las personas } \\
\hline B.1.1 & $\begin{array}{l}\text { Se organizan jomadas de puertas abiertas y actividades regulares de apertura a la comunidad no } \\
\text { universitaria. }\end{array}$ & 34.4 & 47.4 & 9.5 & 8.8 \\
\hline B.1.2 & La facultad es accesible para todas las personas. & 31.2 & 48.4 & 18.2 & 2.1 \\
\hline B.1.3 & Se revisan los recursos para que respondan a una realidad cambiante. & 34.4 & 44.2 & 9.5 & 11.9 \\
\hline B.1.4 & $\begin{array}{l}\text { El alumnado que ingresa a la facultad recibe una atención que garantiza su preparación para la } \\
\text { vida y el mundo laboral. }\end{array}$ & 33.3 & 51.2 & 10.5 & 4.9 \\
\hline B.1.5 & $\begin{array}{l}\text { Los servicios complementarios que ofrece la facultad (cafetería, deportes, tutorías) se ajustan a } \\
\text { las necesidades de la comunidad educativa. }\end{array}$ & 49.8 & 37.9 & 9.5 & 2.8 \\
\hline B.1.6 & Los nombramientos y las promociones docentes son justas. & 50.9 & 30.2 & 3.9 & 15.1 \\
\hline B.1.7 & Se ayuda al profesorado de nueva incorporación a adaptarse a la facultad. & 30.9 & 43.9 & 5.6 & 19.6 \\
\hline B.1.8 & Cuando el alumno accede a la facultad por primera vez se le ayuda a adaptarse. & 49.8 & 36.8 & 5.3 & 8.1 \\
\hline \multicolumn{6}{|c|}{ B.2. Organizar el apoyo para atender a la diversidad } \\
\hline B.2.1 & $\begin{array}{l}\text { La facultad define las políticas para la atención a la diversidad y responde por su divulgación y } \\
\text { cumplimiento. }\end{array}$ & 23.9 & 49.8 & 7.7 & 18.6 \\
\hline B.2.2 & $\begin{array}{l}\text { Se promueve la investigación y formación del profesorado en temas relacionados con la } \\
\text { educación inclusiva. }\end{array}$ & 26.7 & 49.8 & 8.8 & 14.7 \\
\hline B.2.3 & Existen formas de apoyo pedagógico para el alumnado que lo necesita. & 36.8 & 40.7 & 10.2 & 12.3 \\
\hline B.2.4 & Los procedimientos tradicionales de evaluación del alumnado se combinan con otros. & 39.3 & 43.9 & 9.1 & 7.7 \\
\hline B.2.5 & El profesorado recibe formación para gestionar la disciplina en el aula. & 36.5 & 38.9 & 4.6 & 20.0 \\
\hline B.2.6 & Existen alternativas de enseñanza y tutorización no presencial para el alumnado que lo necesita. & 42.5 & 36.5 & 4.9 & 16.1 \\
\hline B.2.7 & Se implementan acciones para detectar las relaciones de abuso de poder o bullying. & 59.6 & 21.1 & 4.6 & 14.7 \\
\hline \multicolumn{6}{|c|}{ C.1. Organizar el proceso educativo } \\
\hline C.1.1 & Los contenidos de las materias de estudio están conectados a problemáticas sociales relevantes. & 20.4 & 60.4 & 14.4 & 4.9 \\
\hline C.1.2 & Los contenidos de las materias de estudio se adecúan a la diversidad del alumnado. & 36.5 & 46.3 & 10.5 & 6.7 \\
\hline C.1.3 & Se organizan grupos de aprendizaje para que todo el alumnado se sienta valorado. & 43.2 & 44.2 & 8.1 & 4.6 \\
\hline
\end{tabular}




\begin{tabular}{|c|c|c|c|c|c|}
\hline C.1.4 & $\begin{array}{l}\text { En la organización de espacios y tiempos se tiene en cuenta a las personas que conforman la } \\
\text { comunidad universitaria. }\end{array}$ & 36.5 & 48.4 & 7.7 & 7.4 \\
\hline C.1.5 & Se implica activamente al alumnado en su propio aprendizaje. & 23.9 & 56.1 & 16.5 & 3.5 \\
\hline C.1.6 & El profesorado apoya el aprendizaje y la participación de todo el alumnado. & 26.3 & 54.7 & 16.1 & 2.8 \\
\hline C.1.7 & El profesorado planifica, revisa y enseña en colaboración con otros docentes. & 32.6 & 46.3 & 9.1 & 11.9 \\
\hline C.1.8 & La evaluación motiva y refleja los logros del alumnado. & 43.9 & 42.8 & 8.4 & 4.9 \\
\hline \multicolumn{6}{|c|}{ C.2. Movilizar recursos } \\
\hline C.2.1 & $\begin{array}{l}\text { Se conocen y se aprovechan los recursos de la comunidad universitaria para asegurar la } \\
\text { inclusión efectiva de todos sus miembros. }\end{array}$ & 35.1 & 44.9 & 6.3 & 13.7 \\
\hline C. 2.2 & Los recursos de la facultad se distribuyen de forma justa para apoyar la inclusión & 44.9 & 32.6 & 7.0 & 15.4 \\
\hline C.2.3 & $\begin{array}{l}\text { La tutoría universitaria es un ámbito integrado en el servicio de orientación de la facultad a } \\
\text { disposición de cualquier miembro de la misma. }\end{array}$ & 27.0 & 47.7 & 7.0 & 18.2 \\
\hline C. 2.4 & La experiencia del profesorado se aprovecha plenamente para enriquecer el proceso educativo. & 27.7 & 51.2 & 13.3 & 7.7 \\
\hline C. 2.5 & El profesorado genera recursos para apoyar el aprendizaje y la participación. & 28.1 & 54.4 & 11.2 & 6.3 \\
\hline C.2.6 & La diversidad del alumnado se utiliza como recurso para enriquecer el proceso educativo. & 33.3 & 48.1 & 12.3 & 6.3 \\
\hline
\end{tabular}

En contraparte, los ítems: Las relaciones entre el profesorado y el alumnado se basan en el respeto mutuo (26.7\%), El alumnado participa en los órganos de representación estudiantil (25.6\%), así como, toda la comunidad universitaria siente orgullo de pertenecer a esta institución $(23.9 \%)$ presentan el mayor nivel de 'totalmente de acuerdo' no sólo en esta sección, sino en todas las demás.

En la segunda sección: Establecer valores inclusivos, estar 'en desacuerdo' se advierte más en el ítem Toda la comunidad educativa se identifica con la filosofía, los principios y los objetivos de la educación inclusiva (44.2\%), sin embargo, también llama la atención varios ítems que necesitan más información, el más alto es $L a$ Facultad implementa acciones para prevenir riesgos psicosociales $(8.8 \%)$.

La tercera sección: Desarrollar una universidad para todas las personas, muestra que los ítems con los que están mayormente 'en desacuerdo' son Los servicios complementarios que ofrece la facultad se ajusta a las necesidades de la comunidad educativa (49.8\%), Los nombramientos y las promociones docentes son justas (50.9\% que, a propósito, es el segundo más alto de toda la escala), así como también Cuando el alumno accede a la facultad por primera vez se le ayuda a adaptarse (49.8\%). Respecto a los aspectos en los que se necesita más información, se encontró dos ítems Se ayuda al profesorado de nueva incorporación a adaptarse a la facultad (19.6\%) y Los nombramientos y las promociones docentes son justas (15.1\%).

La cuarta sección: Organizar el apoyo para atender a la diversidad, tiene ítems que destacan en el indicador 'en desacuerdo', el más alto corresponde a Se implementan acciones para detectar las relaciones de abuso de poder o bullying $(59.6 \%$ que, a propósito, es el porcentaje más alto de toda la escala), pero además necesitan más información para responder al ítem El profesorado recibe formación para gestionar la disciplina en el aula (20\%), así como para el ítem La facultad define las políticas para la atención a la diversidad y responde por su divulgación y cumplimiento (18.6\%).

La quinta sección: Organizar el proceso educativo, muestra que, casi la mitad de los estudiantes están 'en desacuerdo' en al menos dos ítems como: Se organizan grupos de aprendizaje para que todo el alumnado se sienta valorado (43.2\%) y La evaluación motiva y refleja los logros del alumnado (43.9\%). En el ítem que mayor necesidad de información tienen es El profesorado planifica, revisa y enseña en colaboración con otros docentes $(11.9 \%)$.

La última sección: Movilizar recursos, presenta un alto porcentaje de 'en desacuerdo' para el ítem Los recursos de la facultad se distribuyen de forma justa para apoyar la inclusión (44.9\%). En tanto que, los ítems que demandan más información son: La tutoría universitaria es un ámbito integrado en el servicio de orientación de la facultad a disposición de cualquier miembro de esta (18.2\%) y Los recursos de la facultad se distribuyen de forma justa para apoyar la inclusión (15.4\%).

\subsection{Comparación entre secciones y dimensiones}

Para comparar los resultados de las seis secciones, se empleó la prueba Kruskal-Wallis $\left(\chi^{2}=73.13,5\right.$ gl (grados de libertad), $\mathrm{p}=0.000$ ), con la cual se determinó que existen diferencias entre las secciones. Sin embargo, con esta prueba no es posible identificar cuáles son las diferencias que existen entre las secciones, por ello, como una forma de análisis post hoc, se empleó la prueba no paramétrica U de Mann-Whitney. Esta prueba fue empleada para comparar cada sección con todas las demás. Estos resultados permitieron conocer que la sección A.1. Construir comunidad es la más alta (mediana $=1.82$ ) y es diferente significativamente a todas las demás $(\mathrm{Z}<-1.96)$, mientras que, a nivel intermedio se encuentran las secciones C.2. Movilizar recursos (mediana=1.75), C.1. Orquestar el proceso educativo (mediana=1.75) y A.2. Establecer valores inclusivos (mediana=1.86). En contraparte, de forma significativamente inferior a las señaladas $(\mathrm{Z}<-1.96)$, se encontró que las secciones más bajas son B.2. Organizar el apoyo para atender a la diversidad (mediana=1.57) y B.1. Desarrollar una universidad para todas las personas (mediana=1.63). Los valores de las medianas se expresan el diagrama de caja y bigotes (Fig. 1).

Para obtener un indicador para cada una de las tres dimensiones, se promediaron las secciones de este modo A: Crear culturas inclusivas (Media de A.1. Construir comunidad y A.2. Establecer valores inclusivos), B: Elaborar políticas inclusivas (Media de B.1. Desarrollar una universidad para todos y B.2. Organizar el apoyo para atender a la diversidad), y C: Desarrollar prácticas inclusivas (que contiene Media de C.1. Orquestar el proceso educativo y C.2. Movilizar recursos). Estos tres promedios se compararon con la prueba de KruskalWallis, la misma que permitió identificar las diferencias 
significativas entre estas tres secciones $\left(\chi^{2}=42.96,2 \mathrm{gl}\right.$, $\mathrm{p}=0.000)$. En efecto, la dimensión A: Crear culturas inclusivas (mediana $=1.85$ ) es la más alta, seguida de la dimensión C: Desarrollar prácticas inclusivas (mediana=1.73) y, por último, se ubicó la dimensión B: Elaborar políticas inclusivas (mediana=1.60).

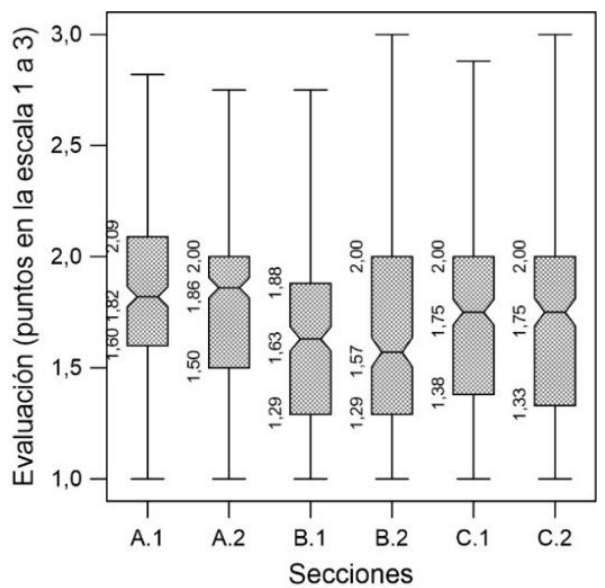

Figura 1. Diagrama de caja y bigotes de las seis secciones.

\subsection{Comparación entre el comportamiento de respuesta de profesores y estudiantes}

Para establecer diferencias entre profesores y estudiantes se empleó el estadístico de prueba U de Mann-Whitney que permitió establecer la existencia de diferencias significativas entre las secciones. La sección A.1. Construir comunidad muestra una mediana mayor en profesores (mediana $=1.82$ ) con respecto a estudiantes (mediana=2.00; $\mathrm{Z}=-3.604$ ), de igual manera, la sección A.2. Establecer valores inclusivos $(\mathrm{Z}=-3.444)$, los estudiantes tienen una mediana de $1.75 \mathrm{y}$ los profesores de 2.00, la sección B.1. Desarrollar una universidad para todas las personas, muestra diferencias significativas entre estudiantes (mediana $=1.50)$ y profesores (mediana=1.88; $\mathrm{Z}=-4.140$ ), ello no ocurre en la sección B.2. Organizar el apoyo para atender a la diversidad $(\mathrm{Z}=-$ 1.433) en la cual los estudiantes tienen una mediana de 1.57 y los profesores de 1.71. La sección C.1. Orquestar el proceso educativo también tiene diferencias entre profesores (mediana $=1.73$ ) y estudiantes (mediana $=2.00$; $\mathrm{Z}=-3.177$ ), de igual manera en la sección C.2. Movilizar recursos $(\mathrm{Z}=-3.816)$ en la que los docentes tienen una mediana de 1.67 y los profesores de 2.00 . El valor de las medianas del profesor comparado con el estudiante se aprecia en el diagrama de caja y bigotes (Fig. 2). En definitiva, con excepción de la sección B.2, en todos los casos se advierte que los profesores tienen una percepción más alta de la inclusión educativa que los estudiantes.

Para resumir, a nivel general, la inclusión educativa en la Facultad de Psicología de la Universidad de Cuenca, se comparó la situación desde la perspectiva de estudiantes y profesores. Según la prueba U de Mann-Whitney, se advierte que existe diferencia significativa en la dimensión A: Crear culturas inclusivas ( $\mathrm{Z}=-4.147$, $\mathrm{p}=0.000$ ) pues la mediana de los profesores es de 2.05 y la de los estudiantes de 1.82. Con esta prueba también se estableció diferencias en la dimensión B: Desarrollar prácticas inclusivas $(\mathrm{Z}=-2.993, \mathrm{p}=0.003)$ según la cual la mediana de los estudiantes es de 1.58 y la de los docentes de 1.79. Por último, la dimensión C: Elaborar políticas inclusivas demostró distribución normal en sus datos por lo que se empleó la prueba $t$ de Student para muestras independientes en la que también se advirtió diferencias significativas $(\mathrm{t}(283 \mathrm{gl})=-4.22, \mathrm{p}=0.000)$. En definitiva, existe mayor discrepancia entre estudiantes y docentes en la dimensión $\mathrm{A}$, luego en la $\mathrm{C}$ y por último en la $\mathrm{B}$.

\section{DISCUSIÓN}

La educación inclusiva pretende ofrecer educación de calidad para todos, respetando la diversidad, luchando contra la desigualdad social y promoviendo una cultura inclusiva (Leire et al., 2010). En los últimos años, dentro del contexto universitario, encontramos aulas heterogéneas caracterizadas por una diversidad de estudiantes, este escenario invita a analizar sobre los factores que influyen en las IES para que sean consideradas espacios inclusivos. En este proceso de análisis, resulta importante reflexionar sobre las percepciones de los distintos actores de la comunidad

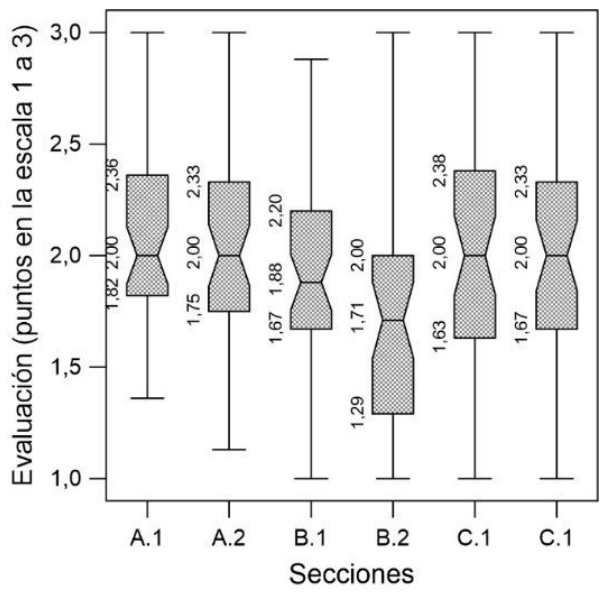

Profesores

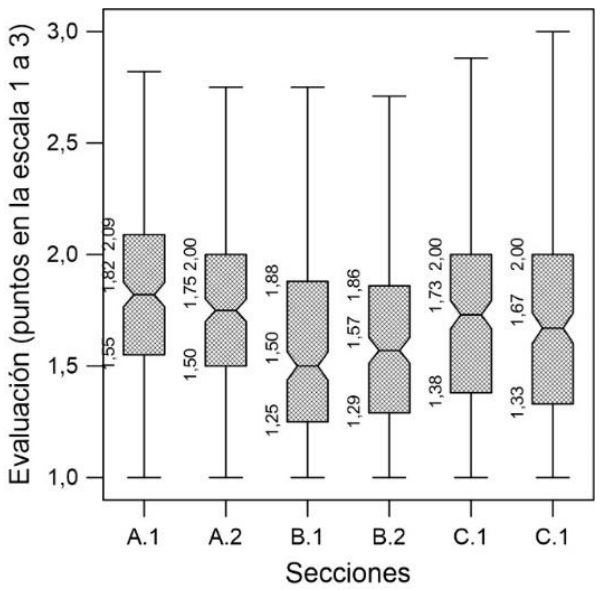

Estudiantes

Figura 2. Diagrama de caja y bigotes de las seis secciones según profesores y estudiantes. 
universitaria relacionados con culturas, políticas y prácticas inclusivas, para establecer elementos que se puedan mejorar contrastando puntos de vista de los distintos representantes del contexto universitario.

La filosofía de la inclusión basada en la diversidad implica una visión diferente de la educación, aquí el énfasis recae en desarrollar una educación que valore y respete la diferencia, donde el progreso de los estudiantes dependa del tipo de oportunidades y apoyos que se les brinde, de la generación, desarrollo y creación de políticas, prácticas y culturas inclusivas (Echeita \& Navarro, 2014; Booth et al., 2015) en cada una y al interior de todas las IES. En esta investigación la percepción de docentes y estudiantes sobre la dimensión A: Crear culturas inclusivas, tiene la percepción más alta de la facultad (mediana de 1.85 puntos) lo que permite distinguir que, docentes y estudiantes están motivados en establecer valores inclusivos, con una tendencia positiva hacia la creación de una cultura inclusiva. Booth \& Ainscow (2002) desde el ámbito escolar, señalan que la cultura se refiere "al desarrollo de valores inclusivos, compartidos por todo el personal de la escuela, los estudiantes, los miembros del Consejo Escolar y las familias" (p.18), y proponen la necesidad de instaurar una comunidad escolar segura, acogedora, colaboradora y estimulante en la que toda persona sea tomada en cuenta y se promuevan valores inclusivos. A nivel universitario, si hablamos de cultura inclusiva podríamos entender que se refiere al conjunto de reglas, símbolos, creencias y valores compartidos por el personal administrativo, estudiantes, docentes y autoridades, por tanto, la cultura inclusiva cambia y se nutre de todos los participantes en ella.

En la sección Construir comunidad, se encuentran percepciones positivas relacionadas con los ítems de la sección Establecer valores inclusivos, aspectos que coincide con lo encontrado por los investigadores Ortiz \& Lobato (2003) en el estudio "Escuela inclusiva y cultura escolar: algunas evidencias empíricas", quienes indican que la cultura y los valores inclusivos radican en poseer un ambiente positivo con adecuadoss niveles de comunicación y respeto entre los integrantes de la comunidad educativa. Por su parte, Márquez \& Sandoval (2019), sostienen que avanzar hacia la construcción de sistemas educativos con una cultura inclusiva no es tarea fácil ni puede improvisarse, la participación de los estudiantes compartiendo espacios, actividades y enseñanzas refuerza actitudes y valores positivos hacia la diversidad. En esta misma línea la UNESCO (2016) señala que para avanzar en la construcción de culturas inclusivas debe facilitarse las condiciones de acceso y participación de todos los estudiantes, sobre todo de aquellos que estén en situaciones de vulnerabilidad y exclusión.

En la misma sección, encontramos, que los docentes reconocen como importante la filosofía, principios y los objetivos de la educación inclusiva para avanzar hacia universidades inclusivas, sin embargo, existe una diferencia significativa en relación con la percepción de los estudiantes en las secciones: Establecer valores inclusivos y Desarrollar una universidad para todas las personas. Al respecto, Márquez \& Sandoval (2019), señalan que los valores de un sistema inclusivo lo constituyen: la libertad, responsabilidad e igualdad de

\footnotetext{
${ }^{2}$ Accesibilidad que de alguna manera está determinada por la Secretaría de Educación Superior, Ciencia, Tecnología e Innovación (SENESCYT), pues a nivel superior la Universidad
}

protección de los derechos, aspectos que al estar reconocidos por los docentes, se convierten en elementos claves para avanzar hacia sistemas universitarios más inclusivos. Otros estudios realizados, no coinciden con estos resultados, Fernández (2013) por ejemplo, en su investigación sobre "Competencias docentes y educación inclusiva" encuentra que los docentes valoran los principios y creencias de lo que se hace y falta por hacer en cuanto a educación inclusiva. En esta sección encontramos también, que tanto docentes como estudiantes observan a la facultad como un espacio que pretende contribuir en la mejora del entorno local y regional, resultados que son similares con lo obtenido por González-Gil, Martín-Pastor, Poy \& Jenaro (2016) en un estudio denominado "Percepciones del profesorado sobre la inclusión" quienes afirman que las actitudes hacia la inclusión que tienen los profesores es muy positiva y genera altas expectativas en todos los estudiantes, sin embargo que muestran resistencia a la hora de modificar sus prácticas educativas para atender la diversidad.

La dimensión "Políticas inclusivas", determinan la responsabilidad que tienen los centros de educación superior de ofrecer a todos los estudiantes los mejores escenarios educativos, políticas que no solo amplíen el acceso, sino que desarrollen medidas que mejoren la permanencia y el éxito universitario de los estudiantes. Contar con políticas inclusivas garantiza a la inclusión como el eje del desarrollo de la institución universitaria, y que, a partir de las mismas, se mejore el aprendizaje y la participación de todo el estudiantado (Márquez \& Sandoval, 2019). En los resultados obtenidos en la sección B.1. Desarrollar una universidad para todas las personas, de acuerdo con la percepción de docentes y estudiantes se percibe una facultad con accesibilidad para todos ${ }^{2}$. Estas derivaciones son una aproximación a la compleja relación que se establecen entre las políticas inclusivas y las actitudes hacia la inclusión de todos los integrantes de la comunidad universitaria. Se hace necesario repensar el rol de cada uno de ellos para generar una sociedad que se reconstruya mirando de frente la diversidad e inclusión de todos, garantice la accesibilidad y disminuya las barreras arquitectónicas y físicas que persisten en las universidades impidiendo la movilidad y participación de todos los estudiantes (Márquez \& Sandoval, 2019).

En cuanto a las prácticas inclusivas, existe una orientación positiva de dichas prácticas, en el ítem organizar el proceso educativo, situación que coincide con Moriña et al. (2015) en su estudio denominado "Educación inclusiva en la enseñanza superior: soñando al profesorado ideal", quienes sostienen que para una apropiada respuesta a la diversidad, los docentes, requieren metodologías claras, preparación y actitudes positivas hacia la inclusión, es decir, para optimizar las prácticas inclusivas deben corregir sus actitudes. Por su parte, Márquez \& Sandoval (2019) indican que las universidades están utilizando el discurso de inclusión para garantizar el acceso a los estudiantes diversos, pero no comprometen sus culturas, políticas y prácticas para apoyar la permanencia y finalización de los estudios, con lo cual se puede demostrar que en la práctica, tenemos una inclusión mal entendida y tergiversada.

establece el número de cupos y la SENESCYT selecciona los aspirantes. 
Los resultados obtenidos en la percepción relacionada con la dimensión desarrollar prácticas inclusivas, permiten sostener que docentes y estudiantes son conscientes de la diversidad y que por tanto, se despliegan una serie de prácticas, como organización de grupos de aprendizaje, organización de espacios y tiempos, distribución de los recursos de manera justa, resultados que coinciden con Simón \& Carballo (2019) quienes sostienen que los docentes reclaman a las universidades mayor formación que les ayude a construir entornos más inclusivos. Para estos autores, son los docentes quienes demandan una mayor información sobre los servicios de apoyo, la normativa que regula la universidad y las necesidades de formación sobre el tema. De igual manera, Cotán (2017) en su estudio denominado "Educación inclusiva en las IES: narrativas de estudiantes con discapacidad" señala que gran parte de la comunidad universitaria no conoce si existen normativas que apoyen la inclusión, y si las conocen, no saben si se aplican o no, sin embargo, en las prácticas se despliegan una serie de acciones que buscan garantizar la educación de los estudiantes con diversidad. Por ello, la creación de entornos universitarios inclusivos, donde participe activamente toda la comunidad universitaria, no es una tarea fácil, implica la generación y transformación de políticas, estructuras y prácticas institucionales que permitan aprender a vivir con la diferencia, diversidad que es considerada como un aspecto positivo y que fomenta la cohesión social (Márquez \& Sandoval, 2019).

\section{CONCLUSIÓN}

Generar políticas, prácticas y crear culturas inclusivas dentro de las IES, es una exigencia de la sociedad del siglo XXI en apoyo a la construcción de una sociedad sostenible y de paz. En términos generales, con los datos obtenidos podemos concluir que docentes y estudiantes de la Facultad de Psicología de la Universidad de Cuenca (Ecuador), son conscientes de la importancia de atender la diversidad, están de acuerdo con establecer valores inclusivos dentro de su entorno de trabajo, sin embargo, consideran que la educación inclusiva, a nivel universitario, debe ser un proceso sistemático que inicia con pequeños cambios, para lo cual se requiere la participación e involucramiento de toda la comunidad universitaria.

La mayoría de los docentes consultados, manifiestan no haber recibido formación en educación inclusiva, evidenciando la carencia de información para organizar y planificar los procesos educativos, desde un enfoque de atención a la diversidad, resultado que revela la necesidad de implementar procesos de capacitación y sensibilización sobre temas de inclusión a nivel universitario, lo que seguramente ayudará a reducir barreras, tanto para el aprendizaje y la participación como para la movilidad de los miembros de la comunidad universitaria. Por otro lado, a pesar de que la percepción de estudiantes y docentes de la Facultad de Psicología de la Universidad de Cuenca, de manera general, es que la facultad es accesible a todos y eso los hace sentirse orgullosos de pertenecer a ella, todavía perciben la falta de movilización de recursos y que estos sean distribuidos de manera justa para apoyar la inclusión, no solo enfocándose en el acceso, sino garantizando la permanencia y culminación de la carrera elegida, tal como lo señala la normativa que regenta la ES.

Finalmente, el estudio plantea, en su análisis prospectivo, la posibilidad de realizar un análisis factorial exploratorio en todas las Facultades de la Universidad de Cuenca, con el propósito de confirmar la fiabilidad del instrumento debido a que, varias preguntas ( 8.4 de los ítems) presentan valores inusuales respecto al conjunto en el que están contenidas. De igual manera, se sugiere ampliar la muestra de docentes y estudiantes en toda la universidad, con la finalidad de generalizar resultados sobre percepción de políticas, culturas y prácticas inclusivas a nivel de ES.

\section{AGRADECIMIENTO}

Este estudio se realizó en el marco del proyecto: "Percepción de docentes y estudiantes sobre culturas, políticas y prácticas inclusivas en educación superior" ganador del XVII concurso de proyectos de investigación de la Universidad de Cuenca (DIUC). Expresamos nuestro reconocimiento y agradecimiento a los estudiantes Loja Angélica y Tello Adrián, así como a Jara Ximena y Narváez Alejandro, quienes desde la coordinación del proyecto trabajaron en la recolección de datos, insumos con los que elaboraron sus tesis de graduación.

\section{REFERENCIAS}

Ainscow, M., Booth, T., \& Dyson, A. (2006). Mejorando escuelas, desarrollando inclusión. Londres. https://doi.org/10.4324/9780203967157

Asamblea Nacional. (2010). Ley Orgánica de Educación Superior LOES. Quito, Ecuador: Registro Oficial Suplemento 298, 69 págs. Disponible en https://www.educacionsuperior.gob.ec/wpcontent/uploads/downloads/2014/03/LEY_ORGANI CA_DE_EDUCACION_SUPERIOR_LOES.pdf

Aznar, P., \& Barrón, A. (2017). El desarrollo humano sostenible. Un compromiso educativo. Teoría de la Educación, 29(1), 25-53. http://dx.doi.org/10.14201/teoredu20172912553

Booth, T., \& Ainscow, M. (2002). Guía para la evaluación y mejora de la educación inclusiva. Index for Inclusion. Madrid, España: Consorcio Universitario para la Educación Inclusiva (original en inglés publicado en Bristol: CSIE, 2000).

Booth, T., Simón, C., Sandoval, M., Echeita, G., \& Muñoz, Y. (2015). Index for inclusion. Guía para la educación inclusiva. Promoviendo el aprendizaje y la participación en las escuelas: nueva edición revisada y ampliada. Revista Iberoamericana sobre Calidad, Eficacia y Cambio en Educación, 13(3), 5-19.

Corral, K., Villafuerte, J., \& Bravo, S. (2015). Realidad y perspectiva de la Educación Inclusiva de Ecuador. Percepciones de los Actores Directos al 2014. Acaraju, Brasil: Congreso Iberoamericano de Investigación Cualitativa. Investigação Qualitativa em Educação / Investigación Cualitativa en Educación, 2, 582-587. 
Cotán, A. (2017): Educación inclusiva en las instituciones de educación superior: narrativas de estudiantes con discapacidad. Revista Española de Discapacidad, 5(I), 43-61. https://doi.org/10.5569/2340-5104.05.01.03

De la Hoz Blanco, J. (2017). Estilos de gestión y cultura institucional en las organizaciones escolares. Revista Encuentros, 15(1), 61-75. http://dx.doi.org/10.15665/re.v15i1.611

Durán, D., Echeita, G., Giné, C., López, M.L., Miquel, E., Moratalla, S., \& Sandoval, M. (2002). Guía para la evaluación y mejora de la educación inclusiva. Madrid, España: Consorcio Universitario para la Educación Inclusiva.

Echeita, G., \& Navarro D. (2014). Educación inclusiva y desarrollo sostenible. Una llamada urgente a pensarlas juntas. Edetania, 46, 141-161.

Espinosa, C., Gómez, V., \& Cañedo, C. (2012). ¿Integración o inclusión? La educación superior ecuatoriana y el pleno acceso de estudiantes con discapacidad. Revista Ciencia y Sociedad, 37(3), 1-19.

Fernández, J. (2013). Competencias docentes y educación inclusiva. Revista Electrónica de Investigación Educativa, 15(2), 82-99.

Fernández, N., \& Pérez, C. (2016). La educación superior latinoamericana en el inicio del nuevo siglo. Situación, principales problemas y perspectivas futuras. Revista Española de Educación Comparada, $27,123-148$ htpps://doi.org/10.5944/reec.27.2016.15044

González-Gil, F., Gómez-Vela, M., \& Jenaro, C. (2007). Índex para la Inclusión: Desarrollo del juego, el aprendizaje y la participación en Educación Infantil. Salamanca: Universidad de Salamanca. Disponible en: https://www.eenet.org.uk/resources/docs/Index $\% 20 \mathrm{E}$ Y\%20Spanish.pdf

González-Gil, F., Martin-Pastor, E., Poy, R., \& Jenaro, C. (2016). Percepciones del profesorado sobre la inclusión: Estudio preliminar. Revista Electrónica Interuniversitaria de Formación del Profesorado, 19(3), 11-24. http://dx.doi.org/10.6018/reifop.19.3.219321

González, R., González, B., Guerrero, V., \& Ríos, G. (2018). Principales retos a la inclusión en la educación superior ecuatoriana. Enfermería Investiga, 3, 84-90.

Herdoíza, M. (2015). Construyendo igualdad en la educación superior. Fundamentación y lineamientos para transversalizar los ejes de igualdad y ambiente. Quito, Ecuador: SENESCYT. Disponible en: https://www.educacionsuperior.gob.ec/wpcontent/uploads/downloads/2018/11/Construyendoigualdad-en-la-educacion-superior_nov_2018.pdf

Jara, R., Melero, N., \& Guichot, E. (2015). Inclusión socioeducativa, perspectivas y desafíos: Universidad Politécnica Salesiana del Ecuador y Universidad de Sevilla España. Alteridad. Revista de Educación, 10(2), 164-179. https://doi.org/10.17163/ALT.V10N2.2015.03

Jara, X., \& Narváez, E. (2019). Percepción de docentes universitarios sobre culturas, políticas y prácticas inclusivas en la Facultad de Psicología (Bachelor's tesis). Disponible en: http://dspace.ucuenca.edu.ec/ handle/123456789/32554

Leire, A., Fernández, J., \& Goicoechea, P. (2010). ¿La educación inclusiva como utopía que nos ayuda a caminar? Congreso Iberoamericano de Educación, Educación Inclusiva. Recuperado de http://www.chubut.edu.ar/descargas/secundaria/cong reso/ EDUCINCLUSIVA/R1879_Darreche.pdf

Loja, G., \& Tello, A. (2019). Percepción de los estudiantes de la Facultad de Psicología sobre prácticas, políticas y culturas inclusivas (Bachelor's tesis). Disponible en: http://dspace.ucuenca.edu.ec/ handle/123456789/32431

Márquez, C., \& Sandoval, M. (2019). Claves para promover la inclusión en educación superior. En: Márquez Vázquez (Ed.), Avanzamos hacia Universidades más inclusivas. De la retórica a los hechos. pp. 45-60. Madrid. Editorial DYKINSON, S.L. https://www.researchgate.net/publication/ 334658162

Martínez, R., Castellanos, M. Á., \& Chacón, J. C. (2015). Análisis de datos en Psicología y Ciencias de la Salud. Volumen II: Inferencia Estadística. EOS.

Moriña, A., Cortés, M., \& Molina, V. (2015). Educación inclusiva en la enseñanza superior: soñando al profesorado ideal. Revista Latinoamericana de Educación Inclusiva, 9(2), 161-175.

Naciones Unidas. (2015a). Edición ilustrada de la Declaración Universal de los derechos humanos (DUDH). 1-72. Disponible en: https://www.un.org/es/udhrbook/

Naciones Unidas. (2015b). Transformar nuestro mundo: la Agenda 2030 para el Desarrollo Sostenible. Asamblea General. 1-40. Disponible en: https://unctad.org/meetings/es/SessionalDocuments/a res70d1_es.pdf

Ochoa Cervantes, A. (2019). El tipo de participación que promueve la escuela, una limitante para la inclusión. Alteridad, 14(2), 184-194. https://doi.org/10.17163/alt.v14n2.2019.03

Ortiz, M., \& Lobato, X. (2003). Escuela inclusiva y cultura escolar: algunas evidencias empíricas. XIII Congreso Nacional e Iberoamericano de Pedagogía, 55, 27-39.

Pardo, P. (2011). La función de la universidad en las sociedades del conocimiento. Aula: Revista de Pedagogía de la Universidad de Salamanca, 17, 145158.

Ponce, J., \& Carrasco, F. (2017). Acceso y equidad a la educación superior y posgrado en el Ecuador, un enfoque descriptivo. Mundos Plurales: Revista Latinoamericana De Políticas y Acción Pública, 3(2), 9-22. https://doi.org/10.17141/ mundosplurales.2.2016.2841

Ruxton, G., \& Beauchamp, G. (2008). Time for some a priori thinking about post hoc testing. Behavioral Ecology, 19(3), 690-693. https://doi.org/10.1093/beheco/arn020

Salceda, M., \& Ibáñez, A. (2015). Adaptación del Index for Inclusion al ámbito de la educación superior: Estudio preliminar. Intangible Capital, 11(3), 508545. https://doi.org/10.3926/ic.647 
SENPLADES. (2017). Plan Nacional de desarrollo 2017-2021. "Toda una vida". Secretaría Nacional de Planificación y desarrollo. Quito Ecuador.

Disponible en:

https://www.planificacion.gob.ec/plan-nacional-dedesarrollo-2017-2021-toda-una-vida/

Simón, R., \& Carballo, R. (2019). Educación Inclusiva en la Universidad: el papel del profesorado. En Márquez Vázquez (Ed.), Avanzamos hacia Universidades más inclusivas. De la retórica a los hechos. pp. 100-121. Madrid. Editorial DYKINSON, S.L.

Sinchi, E., \& Gómez, G. (2018). Acceso y deserción en las universidades. Alternativas de financiamiento. Alteridad, 13(2), 274-287.

https://doi.org/10.17163/alt.v13n2.2018.10.
UNESCO. (2016). Educación 2030: Declaración de Incheon y Marco de Acción para la realización del Objetivo de Desarrollo Sostenible 4: Garantizar une educación inclusiva y equitativa de calidad y promover oportunidades de aprendizaje permanente para todos. Organización de las Naciones Unidas para la Educación, la Ciencia y la Cultura. 1-60. Disponible en: https://unesdoc.unesco.org/ ark:/48223/pf0000245656_spa

Universidad de Cuenca. (2017). Instructivo que regula el proceso de Inclusión en la Universidad de Cuenca. Unidad de Bienestar Universitario, 1-4. 